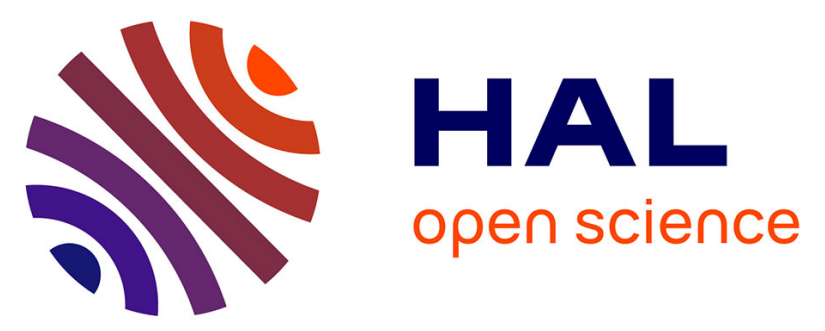

\title{
Effect of the absorption coefficient of aluminium plates on their thermoelastic bending in photoacoustic experiments
}

Dragan Markushev, J Ordonez-Miranda, S Rabasović, S Galovic, M. Todorovic, E. Bialkowski

\section{To cite this version:}

Dragan Markushev, J Ordonez-Miranda, S Rabasović, S Galovic, M. Todorovic, et al.. Effect of the absorption coefficient of aluminium plates on their thermoelastic bending in photoacoustic experiments . Journal of Applied Physics, 2015, 117 (24), pp.245309. 10.1063/1.4922718 . hal-01357610

\author{
HAL Id: hal-01357610 \\ https://hal.science/hal-01357610
}

Submitted on 31 Aug 2016

HAL is a multi-disciplinary open access archive for the deposit and dissemination of scientific research documents, whether they are published or not. The documents may come from teaching and research institutions in France or abroad, or from public or private research centers.
L'archive ouverte pluridisciplinaire HAL, est destinée au dépôt et à la diffusion de documents scientifiques de niveau recherche, publiés ou non, émanant des établissements d'enseignement et de recherche français ou étrangers, des laboratoires publics ou privés. 


\title{
Effect of the Absorption Coefficient of Aluminium Plates on their Thermoelastic Bending in Photoacoustic Experiments
}

D. D. Markushev ${ }^{1 *}$, J. Ordonez-Miranda ${ }^{2}$, M. D. Rabasović ${ }^{1}$, S. Galović ${ }^{3}$, D. M. Todorović ${ }^{4}$, S. E. Bialkowski ${ }^{5}$

${ }^{1}$ Institute of Physics, University of Belgrade, Pregrevica 118, 11080 Belgrade-Zemun, Serbia

${ }^{2}$ Laboratoire d'Énergétique Moléculaire et Macroscopique, Combustion, UPR CNRS 288, Ecole Centrale Paris, Grande Voie des Vignes, 92295 Châtenay Malabry, France.

3 Institute of Nuclear Sciences "Vinca", University of Belgrade, PO Box 522, 11001 Belgrade, Serbia.

${ }^{4}$ Institute for Multidisciplinary Researches, University of Belgrade, P.O.Box 33, 11030 Belgrade, Serbia.

${ }^{5}$ Department of Chemistry and Biochemistry, Utah State University, Logan, UT 84322-0300, USA.

\begin{abstract}
The open-cell photoacoustic signal measured in the transmission configuration for aluminum thin plates with thicknesses of $280 \mu \mathrm{m}, 197 \mu \mathrm{m}$, and $112 \mu \mathrm{m}$ is experimentally and theoretically analyzed, in the $20 \mathrm{~Hz}-7 \mathrm{kHz}$ modulation frequency range. It is shown that the observed differences between the predictions of the standard thermoelastic model and the experiment data of both the amplitude and phase of the photoacoustic signal can be overcome by considering the aluminum samples coated with a thin layer of black paint as volumeabsorber materials. This new approach provides a quite good agreement with the obtained experimental data, in the whole frequency range, and yields an effective absorption coefficient of $(16 \pm 2) \mathrm{mm}^{-1}$, for a $280 \mu \mathrm{m}$-thick sample. The introduction of the finite absorption coefficient led to the correct ratio between the thermal diffusion and thermoelastic components of the photoacoustic signal. Furthermore, it is found that the "volume-absorber" approach accurately describes the behavior of the amplitude, but not that of the phase recorded for a $112 \mu \mathrm{m}$-thick sample, due to its relatively strong thermoeleastic bending, which is not considered by this theory. Within the approximation of the small bending, the proposed "volume-absorber" model provides a reliable description of the photoacoustic signal for Al samples thicker than $112 \mu \mathrm{m}$, and extends the applicability of the classical "opaque" approach.
\end{abstract}


Keywords: Open photoacoustic cell; Absorption coefficient; Thermoelastic bending; High modulation frequency.

PACS: 65.60.+a; 65.80.-g; 65.90.+i

*Corresponding author

email: dragan.markushev @ipb.ac.rs 


\section{INTRODUCTION}

The photoacoustic technique is one of the most useful and reliable photothermal techniques for performing the thermal and optical characterization of a wide variety of materials, because of its high sensitivity and versatility in the nondestructive evaluations of gases, liquids, and solids. ${ }^{1-10}$ The working principle of this spectroscopic technique is based on the photoacoustic effect discovered by Alexander Graham Bell, ${ }^{11}$ who found that an acoustic signal is produced when a sample in an enclosed cell is heated up with light having a periodically varying intensity. Theoretical models to describe this acoustic signal in terms of pressure fluctuations due to thermal expansion and contraction (thermal diffusion TD signal) of a gas layer have been developed by Rosencwaig and Gersho ${ }^{12}$ and by McDonald and Wetsel. ${ }^{13}$ The predictions of these models have been validated with a lot of experimental data reported in the literature for solid and liquids samples. ${ }^{1,2,5,14-18}$ When the solid sample undergoes strong temperature gradient, it also bends and an additional periodic pressure wave (thermoelastic TE signal) shows up in the photoacoustic cell. Furthermore, in semiconductors, the sample mechanical stress induced by the presence of free carrier, generates a third pressure fluctuation named plasma elastic (PE). ${ }^{14-16,19-21}$ The individual contribution of these three components to the total photoacoustic (PA) signal depends on the type of sample material, its thickness, and the modulation frequency.

In many experiments, well-known theoretical models do not describe accurately the individual PA signal components, because of the unsuitable assumptions, approximations and/or experimental conditions. By solving the thermoelastic equations, for a membrane with free borders, Rousset et $a l_{.}{ }^{22}$ calculated analytically the contribution of the thermoelastic bending on the photoacoustic signal and used their results to measure the thermal diffusivity of metallic samples. This theoretical approach has shown to be highly useful for materials in which the thermoelastic-induced vibrations can be considered very small. ${ }^{2,4,17,23}$ However, this model ${ }^{22}$ breaks down for flexible materials, with strong thermoelastic bending, as is the case of $\mathrm{Al}$ and semi-transparent materials like glasses. ${ }^{24,25,26}$ Recently, Sommer et al. ${ }^{27}$ found sizeable differences between Rousset et al. ${ }^{22}$ theory and their experimental data obtained for Al samples with thicknesses between $300 \mu \mathrm{m}$ and $890 \mu \mathrm{m}$. This drawback of the theoretical model was overcome with the introduction of an empirical power law to correlate the TD and TE components of the PA signal. Even though this method, ${ }^{27}$ based on the measurement of the thermoelastic bending parameter (fitting parameter) as a function of the sample thickness, 
yields reliable results for the Al thermal diffusivity, it may not retrieve the correct units of each PA signal.

The aim of this paper is to show how the correct ratio between the TD and TE components of the open-cell PA signal can be obtained by treating aluminum plates coated with a relatively thin layer of black paint as a "volume-absorber" material with a given absorption coefficient. This is achieved by matching both the amplitude and phase of the experimental PA signal to the theoretical expression for the pressure fluctuation due to both the TD and TE contributions within the PA cell. The experiments have been performed in the $20 \mathrm{~Hz}-7 \mathrm{kHz}$ modulation frequency range, for three Al samples with thicknesses of $280 \mu \mathrm{m}, 197 \mu \mathrm{m}$, and $112 \mu \mathrm{m}$. By considering that the samples are opaque, clear discrepancies between the Rousset et al. $^{22}$ model and our experimental data are observed for modulation frequencies higher than $1 \mathrm{kHz}$.

\section{THEORETICAL MODEL}

Let us consider a circular and homogeneous plate of thickness $L$ and radius $R_{s}$, such that $l<<R_{s}$ (thin plate), as shown in Fig.1. When the plate is heated up with a laser beam of modulated intensity $I=I_{0} \exp (-\mathrm{j} \omega t)$, where $\mathrm{j}$ is the imaginary unit, $\omega=2 \pi f, f$ is the modulation frequency, and $t$ is the time; it undergoes thermal diffusion, thermal dilatation, and thermoelastic bending. This latter effect is mainly due to the temperature gradient within the plate. ${ }^{22}$ Considering that the laser illuminates uniformly the surface $x=0$ (azimuthal symmetry), and that its spot (with a radius $5 \mathrm{~mm}$ ) is greater than the plate radius $R_{S}=4 \mathrm{~mm}$; the heat flux mostly propagates along the $x$-axis, mainly (1D process). ${ }^{28,29}$

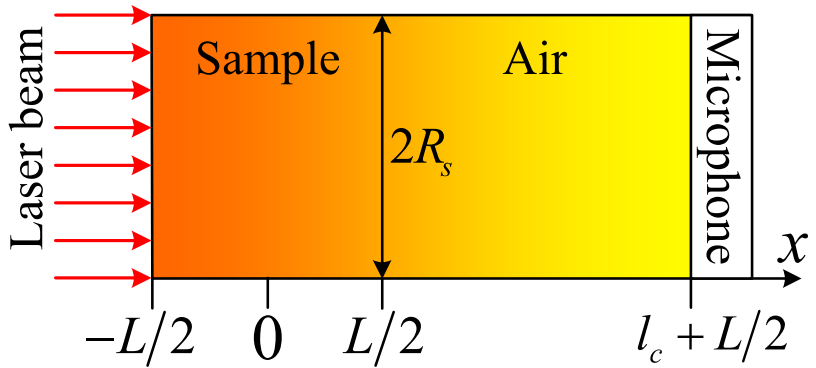

FIG. 1. Scheme of the photoacoustic system under consideration. 
The total PA signal $\partial p_{\text {total }}(\omega)$ detected by the microphone can be obtained as the sum of two independent components, known as the thermal diffusion $\delta p_{\mathrm{TD}}(\omega)$ and the thermoelastic $\delta p_{\text {TE }}(\omega)$ ones: $:^{12,13,23,27,30}$

$$
\delta p_{\text {total }}(\omega)=\left[\delta p_{\mathrm{TD}}(\omega)+\delta p_{\mathrm{TE}}(\omega)\right] \cdot H(\omega)
$$

where $H(\omega)$ is the response function of the system. For small deflections and 1D heat propagation along the $x$-axis, both $\delta p_{\mathrm{TD}}(\omega)$ and $\delta p_{\mathrm{TE}}(\omega)$ components are given by: ${ }^{29}$

$$
\begin{gathered}
\delta p_{\mathrm{TD}}(\omega)=\frac{\gamma_{\mathrm{g}} p_{0} \sqrt{D_{g}}}{l_{c} T_{0} \sqrt{j \omega}} T_{s}(L / 2), \\
\delta p_{\mathrm{TE}}(\omega)=3 \pi \frac{\gamma_{\mathrm{g}} p_{0} \alpha R_{s}^{4}}{L^{3} V_{0}} \int_{-L / 2}^{L / 2} x T_{s}(x) d x,
\end{gathered}
$$

where $T_{0}$ and $p_{0}$ are the ambient temperature and pressure, respectively; $l_{c}$ is the cell length, $\gamma_{g}$ is the ratio of heat capacities at constant pressure and constant volume of the gas (air) within the cell, $D_{g}$ is its thermal diffusivity, $\alpha$ is the coefficient of linear expansion of the sample, and $T_{s}(x)$ is the temperature field at the position $x$ within the sample (Fig. 1). Based on the absorption coefficient $\beta$ of the sample and its thickness $L$, we can define it as optically opaque $(\beta L \rightarrow \infty)$ or as optically semi-transparent $(\beta L>0)$. Semi-transparency means that light is absorbed and heat is generated within the sample volume. In this regard, semi-transparent samples can be considered as volume-absorbers. By solving the Fourier's law of heat conduction, the temperature field $T_{s}(x)$ can be straightforwardly calculated for both cases (see appendix A), and the final results are:

- Opaque sample $(\beta L \rightarrow \infty)$ :

$$
T_{s, a}(x)=\frac{I_{0}}{k_{s} \sigma_{s}} \frac{\cosh \left[\sigma_{s}(L / 2-x)\right]}{\sinh \left(\sigma_{s} L\right)} .
$$

where $I_{0}$ is the laser intensity, $\sigma_{s}=(1+j) \mu_{s}^{-1}$ is the complex wave vector of heat diffusion, $\mu_{s}=\sqrt{D_{s} /(\pi f)}$ is the thermal diffusion length, and $D_{s}$ is the thermal diffusivity of the sample. After inserting Eq. (3) into Eqs. (2a) and (2b), one obtains 


$$
\begin{gathered}
\delta p_{\mathrm{TD}}(\omega)=\frac{a I_{0}}{k_{s} \sigma_{s}} \frac{1}{\sinh \left(\sigma_{s} L\right)}, \\
\delta p_{\mathrm{TE}}(\omega)=\frac{b I_{0}}{2 k_{s}\left(\sigma_{s} L\right)^{2}} J_{1}
\end{gathered}
$$

where $a=\gamma_{\mathrm{g}} p_{0} \sqrt{D_{g}} /\left(l_{c} T_{0} \sqrt{j \omega}\right), b=3 \pi \gamma_{\mathrm{g}} p_{0} \alpha R_{s}^{4} / V_{0}$, and

$$
J_{1}=-1+\frac{2}{\sigma_{s} L} \tanh \left(\sigma_{s} L / 2\right) .
$$

Equations (4) and (5) show that the thermal diffusion and thermoelastic components of the PA signal are both determined by the ratio $L / \mu_{s}$ and they agree with previous results. ${ }^{23}$ For a thermally thin sample $\left(L / \mu_{s}<<1\right)$, Eqs. (4a) and (4b) reduce to

$$
\begin{gathered}
\delta p_{\mathrm{TD}}(f) \approx \frac{a I_{0}}{k_{s} L \sigma_{s}^{2}} \propto f^{-3 / 2}, \\
\delta p_{\mathrm{TE}}(f) \approx \frac{b I_{0}}{24 k_{s}} \propto f^{0},
\end{gathered}
$$

which indicate that, in this regime, the thermoelastic component becomes independent of the modulation frequency $f$, while the thermal diffusion one exhibits the dependence $f^{-3 / 2}$. On the other hand, for a thermally thick sample $\left(L / \mu_{s}>>1\right)$, Eqs. (4a) and (4b) take the form

$$
\begin{gathered}
\delta p_{\mathrm{TD}}(f) \approx \frac{2 a I_{0}}{k_{s} \sigma_{s}} e^{-\sigma_{s} L} \propto \frac{e^{-(1+j) L / \mu_{s}}}{f}, \\
\delta p_{\mathrm{TE}}(f) \approx-\frac{b I_{0}}{2 k_{s}\left(\sigma_{s} L\right)^{2}} \propto f^{-1} .
\end{gathered}
$$

It is therefore clear that at high frequency, such that $\left(L / \mu_{s}>>1\right)$, the thermoelastic contribution is dominant and varies inversely proportional to the frequency.

- Volume-absorber sample $(\beta L>0)$ :

$$
T_{s, b}(x)=\frac{I_{0}}{k_{s} \sigma_{\mathrm{s}}} \frac{1}{1-\left(\sigma_{s} / \beta\right)^{2}}\left\{\frac{\cosh \left[\sigma_{\mathrm{s}}(L / 2-x)\right]-e^{-\beta L} \cosh \left[\sigma_{\mathrm{s}}(L / 2+x)\right]}{\sinh \left(\sigma_{\mathrm{s}} L\right)}-\frac{\sigma_{\mathrm{s}}}{\beta} e^{-\beta(L / 2+x)}\right\},
$$

The combination of Eq. (2) and (8) yields 


$$
\begin{gathered}
\delta p_{\mathrm{TD}}(\omega)=\frac{a I_{0}}{k_{s} \sigma_{s}} \frac{1}{1-\left(\sigma_{s} / \beta\right)^{2}}\left[\frac{1}{\sinh \left(\sigma_{s} L\right)}-\left(\frac{1}{\tanh \left(\sigma_{s} L\right)}+\frac{\sigma_{s}}{\beta}\right) e^{-\beta L}\right], \\
\delta p_{\mathrm{TE}}(\omega)=\frac{b I_{0}}{2 k_{s}\left(\sigma_{s} L\right)^{2}} \frac{1}{1-\left(\sigma_{s} / \beta\right)^{2}}\left[\left(1+e^{-\beta L}\right) J_{1}-\left(\frac{\sigma_{s}}{\beta}\right)^{2} \frac{J_{2}}{\beta L}\right],
\end{gathered}
$$

where $J_{1}$ is given in Eq. (5) and

$$
J_{2}=2-\beta L-(2+\beta L) e^{-\beta L} .
$$

In deriving Eq. (8), we have assumed that when the plate is illuminated at the surface $x=0$, the photonic energy of the laser beam is totally converted into heat within the sample, such that its intensity is well described by the Beer-Lambert law, ${ }^{12}$ which has an exponential decay nature. Note that for thermally thick samples ( $L>>\mu_{s}$, case of high frequency), the thermoelastic component decays as $\delta p_{\mathrm{TE}} \propto\left(\mu_{s} / L\right)^{2}$; but is higher than the thermal diffusion one, which tends to zero $\left(\delta p_{\mathrm{TD}} \rightarrow 0\right)$. On the other hand, the PA signal of thermally thin samples ( $L<<\mu_{s}$, case of low frequency) is predominantly generated by the thermal diffusion component $\delta p_{\mathrm{TD}} \propto \mu_{s} / L$, while the thermoelastic one $\delta p_{\mathrm{TE}}$ keeps smaller and independent of the frequency. Therefore, even though the bending of the sample is always present for low and high frequencies, its relative contribution is minimal for thermally thin samples, which is consistent with our previous results. ${ }^{29}$ Furthermore, for an optically thick sample ( $\beta L>>1$ ), Eqs. (8), (9a), and (9b) reduce respectively to Eqs. (3), (4a), and (4b); as expected. By contrast, for an optically thin sample $(\beta L<<1)$, Eqs. (9a) and (9b) become

$$
\begin{gathered}
\delta p_{\mathrm{TD}}(\omega)=\frac{a I_{0}}{k_{s} \sigma_{s}} \frac{1}{1-\left(\sigma_{s} / \beta\right)^{2}}\left[\frac{1}{\sinh \left(\sigma_{s} L\right)}-\frac{1}{\tanh \left(\sigma_{s} L\right)}-\frac{\sigma_{s}}{\beta}\right], \\
\delta p_{\mathrm{TE}}(\omega)=\frac{b I_{0}}{k_{s}} \frac{1}{1-\left(\sigma_{s} / \beta\right)^{2}}\left[\frac{J_{1}}{\left(\sigma_{s} L\right)^{2}}+\frac{1}{12}\right] .
\end{gathered}
$$

For a sample thermally thick $\left(L / \mu_{s}>>1\right)$ and optically thin $(\beta L<<1)$, the two signals in Eqs. (11a) and (11b) become independent of the sample thickness $L$ and they reduce to

$$
\begin{gathered}
\delta p_{\mathrm{TD}}(f)=\frac{a I_{0} \beta \mu_{s}^{2}}{2 j k_{s}} \propto \beta f^{-3 / 2}, \\
\delta p_{\mathrm{TE}}(f)=-\frac{b I_{0}}{24 j k_{s}}\left(\beta \mu_{s}\right)^{2} \propto \beta^{2} f^{-1} .
\end{gathered}
$$


which explicitly show that the effects of the absorption coefficient are weighted by the modulation frequency, through the product $\beta \mu_{s}$. On the other hand, for a thermally $\left(L / \mu_{s}<<1\right)$ and optically $(\beta L<<1)$ thin sample, Eqs. (11a) and (11b) take the form

$$
\begin{gathered}
\delta p_{\mathrm{TD}}(f)=-\frac{a I_{0}}{k_{s}} \frac{\beta \mu_{s}^{2}}{\left(\beta \mu_{s}\right)^{2}-2 j} \propto \frac{\beta}{\beta^{2}-2 \pi j f / D_{s}}, \\
\delta p_{\mathrm{TE}}(f)=\frac{j b I_{0}}{60 k_{s}} \frac{(\beta L)^{2}}{\left(\beta \mu_{s}\right)^{2}-2 j} \propto \frac{\beta^{2} f}{\beta^{2}-2 \pi j f / D_{s}} .
\end{gathered}
$$

In this case, Eq. (13b) shows that the effect of the absorption coefficient $\beta$ on the thermoelastic component is weighted not only by the diffusion length $\mu_{s}$, as is the case of the thermal diffusion one in Eq. (13a), but also by the sample thickness $L$.

\section{EXPERIMENTAL SETUP}

The experimental setup of the open photoacoustic cell used in the transmission configuration to measure the PA signal is shown in Fig. 2 and explained in detail somewhere else. ${ }^{28,29}$ The sample, held by a rubber O-ring with a $5 \mathrm{~mm}$ outer radius, is directly mounted on an electret microphone front (ECM 30B, Jin In Electronic Co., Ltd) and fixed with vacuum grease to prevent air leaking from the PA chamber. The sample is then illuminated with a red laser diode $(660 \mathrm{~nm})$ whose intensity is modulated with the homemade current modulator within the $20 \mathrm{~Hz}-7 \mathrm{kHz}$ frequency range. Within this frequency range, the effect of roughness of the illuminated surface is expected to be weak ${ }^{29,31}$ and therefore it is not going to be considered in this work. The average laser power is $15 \mathrm{~mW}$ and the laser spot with a $5 \mathrm{~mm}$ radius is big enough to homogeneously illuminate the samples and minimize the $3 \mathrm{D}$ effects. This laser intensity is low enough to neglect the nonlinear effects of the electret microphone, within the full range of modulation frequencies under consideration. ${ }^{1,29}$ The corresponding $\mathrm{Al}$ absorption coefficient at this wavelength of the laser is $\beta=145 \times 10^{3} \mathrm{~mm}^{-1} .^{32-34}$ The resulting amplitude and phase of the PA signal are measured as a function of the modulation frequency by means of a PC sound card (Intel $82801 \mathrm{Ib} / \mathrm{ir} / \mathrm{ih}$ hd audio controller) instead of a lock-in amplifier. This card is a low-cost and portable instrument that is able to record a photoacoustic signal as accurate as the one provided by a lock-in amplifier, as we showed previously, ${ }^{28}$ for the whole range of modulation frequencies analyzed in the present work. All measurements are performed at room temperature (about $20^{\circ} \mathrm{C}$ ). 


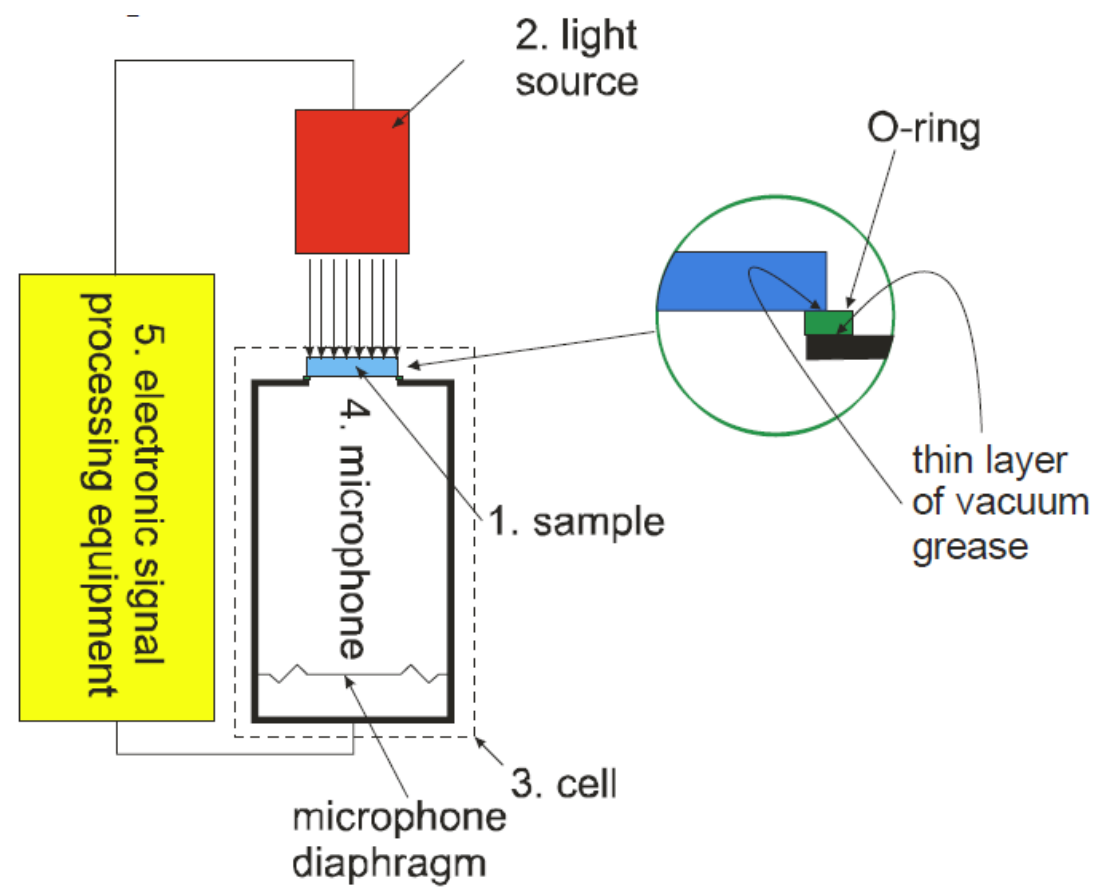

FIG. 2. Scheme of the open-cell photoacoustic setup used in our experiments.

PA measurements have been conducted for three Al plates, which have been cut with a radius $R_{s}=4 \mathrm{~mm}$, and thicknesses $L=280 \mu \mathrm{m}, 197 \mu \mathrm{m}$, and $112 \mu \mathrm{m}$ (with a relative error of about $1 \%$ ), from a commercial aluminum billet. The bulk thermal conductivity, thermal diffusivity, density, specific heat capacity, and coefficient of linear expansion of the samples are: ${ }^{27,32-34}$ $k_{s}=210 \mathrm{~W} \cdot \mathrm{K}^{-1} \cdot \mathrm{m}^{-1}, D_{s}=8.6 \times 10^{-5} \mathrm{~m}^{2} \cdot \mathrm{s}^{-1}, \rho=2700 \mathrm{~kg} \cdot \mathrm{m}^{-3}, c=900 \mathrm{~J} \cdot \mathrm{kg}^{-1} \cdot \mathrm{K}^{-1}$, and $\alpha=23.1 \times 10^{-5} \mathrm{~K}^{-1}$, respectively. Furthermore, to reduce the high reflectivity and improve the optical absorption of the Al samples, ${ }^{35}$ their illuminated surface has been coated with a layer of black pigment, whose uniform thickness is within the micrometer resolution of $4 \mathrm{~m}$, which is quite small in comparison to the one of the samples. This value stands for the average coating thickness measured with a micrometer at the center and edges of the samples. This black paint enhances significantly the rate of light-to-heat conversion within the painting and the first atomic layers underneath the illuminated surface of $\mathrm{Al}$, which act as heat sources. This is why the (paint $+\mathrm{Al})$ samples are going to be considered as one-layer volumeabsorber materials with an effective absorption coefficient $\beta_{\text {eff }}$, which accounts for the efficiency of light-to-heat conversion within the samples, and therefore it depends on their optical properties. In view of the high reflectivity of $\mathrm{Al}\left(R_{2}>0.9\right)$ and high absorbance of the black paint $\left(\beta_{1} l_{1}>1\right)$, the effective absorption coefficient is expected to be smaller than the one of the Al plate $\left(\beta_{\text {eff }}<\beta_{2}\right)$, as demonstrated in appendix II. Taking into account the long 
thickness and high Young's modulus of the Al plate with respect to the corresponding values of the black paint, the thermoelastic bending of the (Al+paint) sample is expected to be determined by the Al plate mainly, and it can therefore be considered as a single layer with an effective absorption coefficient. Furthermore, in this limit of relatively long Al thickness, the presence of the black paint does not modify significantly the heat diffusion through the $\mathrm{Al}$ layer, as shown by Mansanares et $a l^{4}$

\section{RESULTS AND DISCUSSION}

The amplitude $A(f)$ and phase $\varphi(f)$ of the experimental ( $\delta p_{\exp }$ ) and theoretical ( $\delta p_{\text {total, a }} ; \delta p_{\text {total, b }} ; \delta p_{\text {total, b }}^{\prime}$ ) PA signals determined for the Al plate with thickness $L_{1}=280 \mu \mathrm{m}$ are shown in Figs. 3(a) and 3(b), respectively; as a function of modulation frequency $f$. The curves corresponding to $\delta p_{\text {total }, a}$ and $\delta p_{\text {total, b }}$ have been determined by fitting the theoretical models for the totally opaque (Eq. (3a)) and volume-absorber (Eq. (3b)) samples, respectively; to the experimental data associated with $\delta p_{\exp }$. Note that for modulation frequencies higher than $200 \mathrm{~Hz}$, both $A(f)$ and $\varphi(f)$ of $\delta p_{\exp }$ differ from their corresponding theoretical predictions obtained for opaque samples $\left(\delta p_{\text {total }, a}\right)$. This discrepancy was also found for $\mathrm{Al}$ samples thinner than $1 \mathrm{~mm}$, by other authors, ${ }^{27}$ who figured it out by replacing the theoretically predicted $L^{3}$ term in Eq.(2b) by the empirical $L^{2.8}$ power law in the TE component. On the other hand, the quite good agreement between the proposed volume-absorber model ( $\delta p_{\text {total, b }}$ ) with the experimental values of both $A(f)$ and $\varphi(f)$, within the whole range of frequencies, indicates that the introduction of empirical power laws is not required, provided that the (paint+Al plate) sample is treated as a volumeabsorber material with an effective absorption coefficient $\beta_{\text {eff }}=(16 \pm 2) \mathrm{mm}^{-1}$. This effective parameter has been determined by a fitting procedure and is smaller than the one of pure aluminum, as expected. According to Figs. 3(a) and 3(b), this single value of the absorption coefficient permits the correct description of both the amplitude and phase, which shows that the volume-absorber approach is consistent. Note that the inverse of the effective absorption coefficient $\beta_{\text {eff }}^{-1}=63 \mu \mathrm{m}$ is smaller than the total thickness $284 \mathrm{~m}$ of the (paint+Al) sample, which indicates that all the photonic energy of the laser is converted into heat within the sample, as assumed to develop our theoretical model. This reasonable treatment allows us extending the application of Rousset et al. ${ }^{22}$ model for the thermoelastic TE component of the PA signal, to this type of materials. The amplitude and phase of the corrected PA signal $\delta p_{\text {total }, b}^{\prime}$ shown in Figs. 3(a) and 3(b) are obtained by removing all the effects of the response function $(H)$ of our measurement system ${ }^{28}$ and they are derived from

$$
\delta p_{\text {total }, b}^{\prime}(\omega)=\delta p_{\mathrm{TD}, b}^{\prime}(\omega)+\delta p_{\mathrm{TE}, b}^{\prime}(\omega),
$$


which represents the sample PA characteristics, in absence of particular features of our experimental setup. The signal $\delta p^{\prime}{ }_{\text {total }, b}$ has been determined dividing the experimental PA signal by $H$, which was calculated by using a Si thin plate, as detailed in our previous work. ${ }^{29}$ At low frequencies $(f<200 \mathrm{~Hz})$, the deviations of $\delta p_{\text {total, } b}^{\prime}$ with respect to $\delta p_{\text {total, } b}$ are attributed to an impedance mismatch between the microphone and the sound card used instead of a lock-in amplifier, for processing the PA signal. By contrast, at high frequency $(f>1000 \mathrm{~Hz})$, the detected differences are mainly due to the particular characteristics of the electro-acoustic detector. The good agreement between the amplitude and phase of $\delta p_{\text {total, } b}$ $\delta p_{\text {total }, b}$ at intermediate frequencies indicates that our measurement system has a good response in this frequency range.
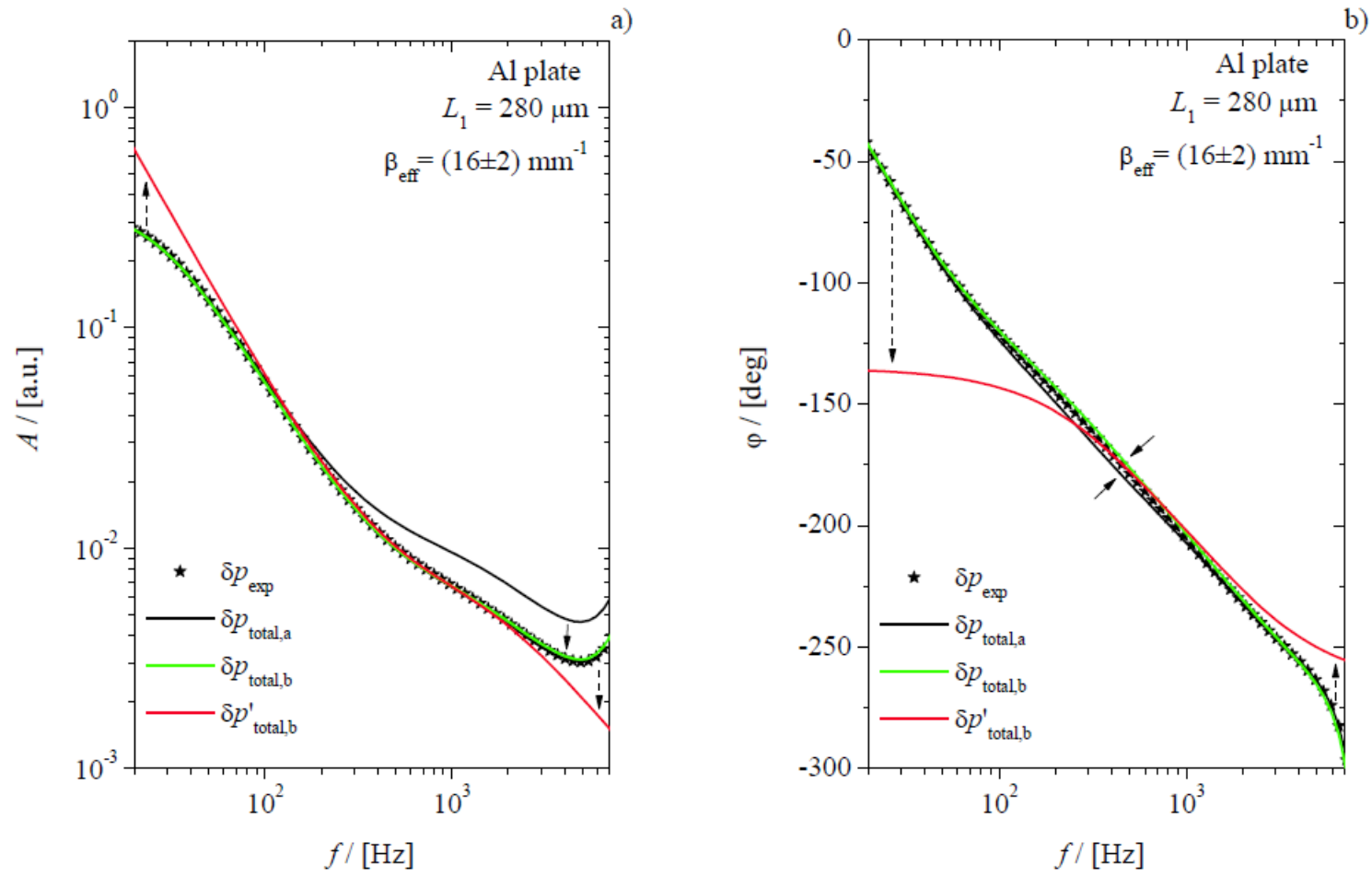

FIG. 3. a) Amplitude $A(f)$ and b) phase $\varphi(f)$ of the PA signal, as a function of modulation frequency $f$ and for the $\mathrm{Al}$ sample thickness $L_{l}=280 \mu \mathrm{m}$. Experimental data $\delta p_{\exp }$ (asterisks),

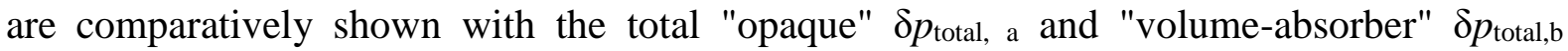
fitting curves along with the corrected $\delta p_{\text {total, } \mathrm{b}}^{\prime}=\delta p_{\text {total, } \mathrm{b}} / H$ signal determined without the instrumental response function $H$ of the measuring system. Levels of corrections are indicated with arrow lines. 
Figures 4 and 5 show the comparison of the experimental and theoretical PA signals obtained for the Al samples of thicknesses $L_{2}=197 \mu \mathrm{m}$ and $L_{3}=112 \mu \mathrm{m}$, respectively. The fitting of the $\delta p_{\text {total,b }}$ PA signal to the experimental data has yielded the following effective absorption coefficients $\beta_{\text {eff }}\left(L_{2}\right)=(17 \pm 2) \mathrm{mm}^{-1}$ and $\beta_{\text {eff }}\left(L_{3}\right)=(20 \pm 2) \mathrm{mm}^{-1}$, which are slightly higher than the previous one determined for the sample thickness $L_{1}=280 \mu \mathrm{m}$. These variations of $\beta_{\text {eff }}(L)$ could be due to the slightly distinct amount (thickness) of black paint on the $\mathrm{Al}$ samples, and the possible presence of tiny air voids within this black coating. As in the case of Fig. 3, the complete agreement between theory and experiment is achieved in both the amplitude $A(f)$ and phase $\varphi(f)$, for the Al sample of thickness $L_{2}=197 \mu \mathrm{m}$ (Fig. 4). For $L_{3}=112 \mu \mathrm{m}$, this concordance still holds in the amplitude but not in the phase. The predictions of the volume-absorber model with the $\beta_{\text {eff }}\left(L_{3}\right)$ correction do not follow the experimental phase and it behaves similarly to the uncorrected one obtained with the "opaque" model. Taking into account that the proposed model is valid for small thermoelastic bending only, its breakdown for thin and strongly bent samples is expected. The present results indicate that this failure occurs for $\mathrm{Al}$ plates with thicknesses equal to or smaller than $112 \mu \mathrm{m}$. Therefore, the description of the PA signal for thinner Al samples requires the generalization of the thermoeleastic model for strong bendings.
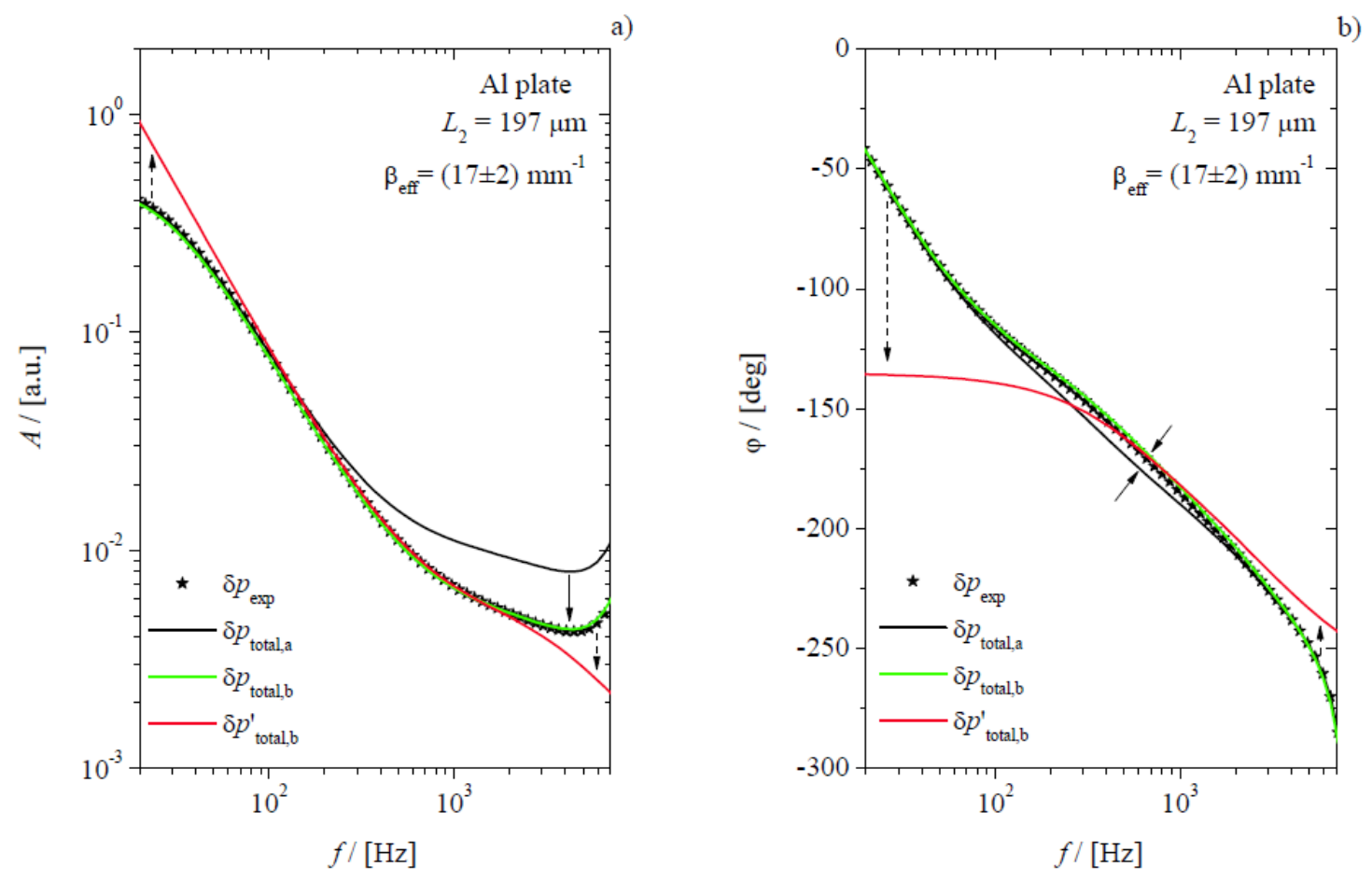

FIG. 4. The same than in Fig. 3, for $L_{2}=197 \mu \mathrm{m}$. 

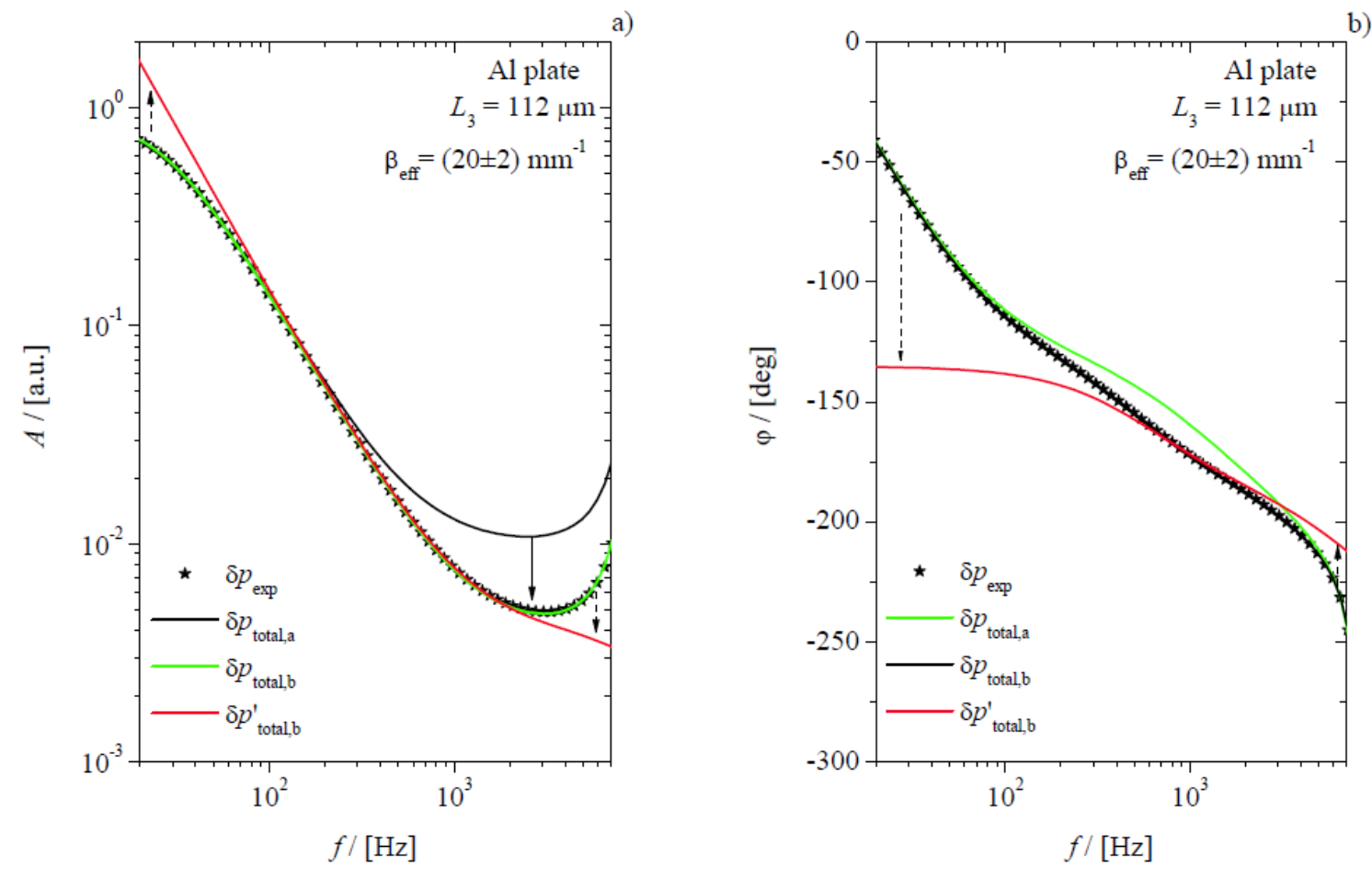

FIG. 5. The same than in Fig. (3), for $L_{3}=112 \mu \mathrm{m}$.

Based on the results shown in Figs. (3)-(5), it is clear that the introduction of $\beta_{\text {eff }}$ in the thermoeleastic model allows us to overcome the differences between the experimental PA signal and the predictions of the "opaque" theory, by accurately describing the behavior of this signal. The effect of the effective absorption coefficient $\beta_{\text {eff }}$ on the TE amplitude (TD phase) is to decrease (increase) its values, in the whole range of modulation frequencies, as shown in Figs. (6) and (7) for the Al samples with thicknesses $L_{1}=280 \mu \mathrm{m}$ and $L_{2}=197 \mu \mathrm{m}$, respectively. Note that the "volume-absorber" model does not change appreciably the frequency dependence of the TE amplitude but rather it displaces it to values lower than those predicted by the "opaque" model. The phase differences predicted by these two models are comparatively smaller than the ones exhibited by the amplitude, and they are present at high frequency $(f>1000 \mathrm{~Hz})$, mainly. The discrepancy between the theoretical predictions of the $\delta p_{\text {total, b }}^{\prime}$ and $\delta p_{\text {total, a }}^{\prime}$ PA signals is hence due to the miscalculation of both the $\delta p_{\mathrm{TD}, a}^{\prime}$ and $\delta p_{\mathrm{TE}, a}^{\prime}$ components of the "opaque" model. It is therefore clear that the proposed "volumeabsorber" model extends the application of the "opaque" one, for samples with small thermoelastic bending. 
a)
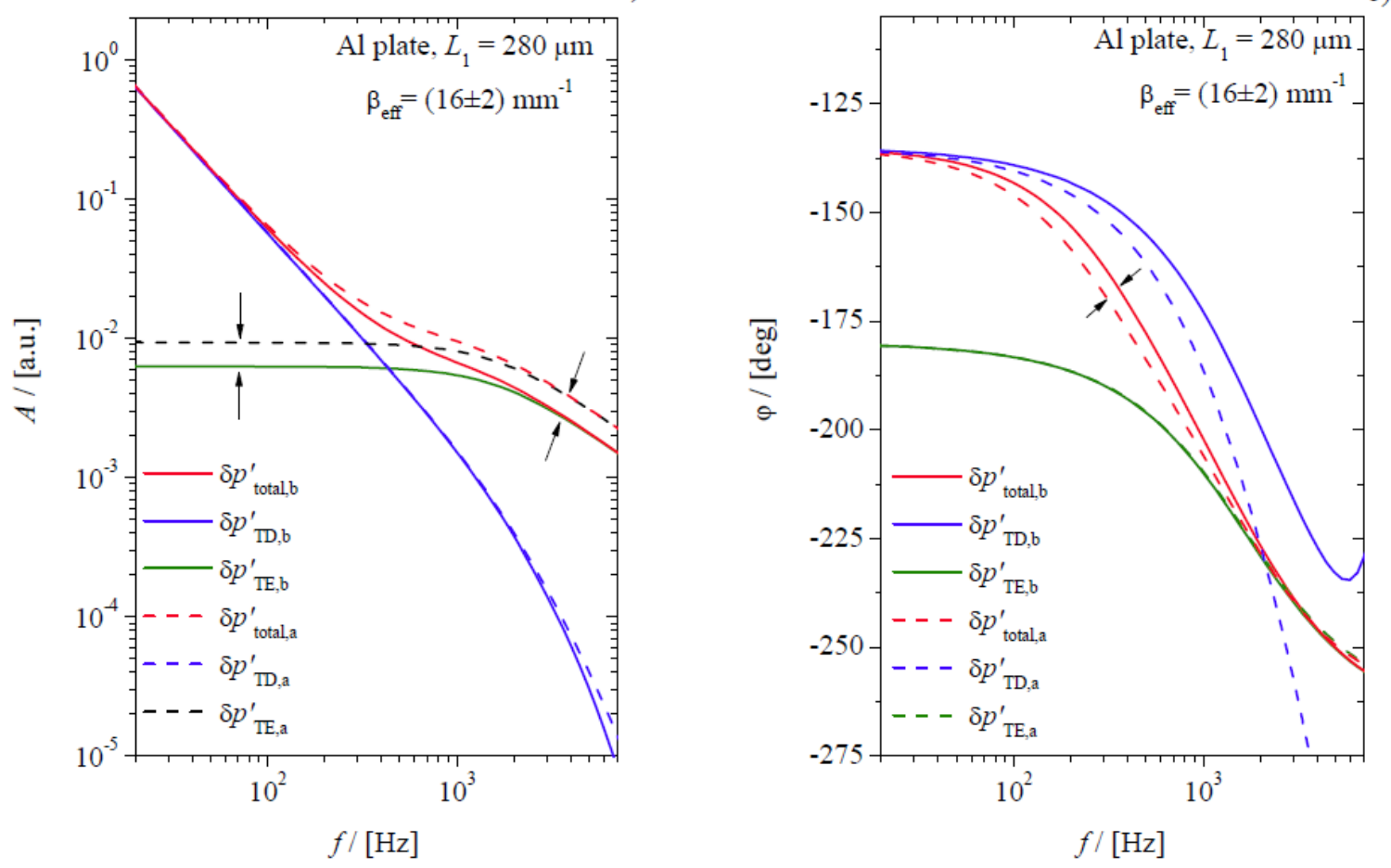

FIG. 6. a) Amplitudes $A(f)$ and b) phases $\varphi(f)$ of the corrected PA signals without the instrumental response of the measuring system, as a function of modulation frequency $f$, for

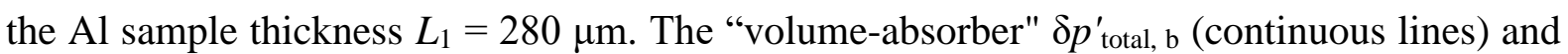

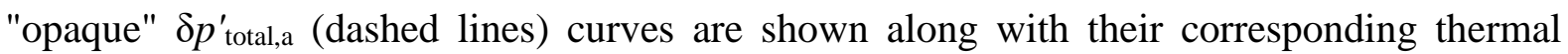
diffusion (TD) and thermoelastic (TE) components.
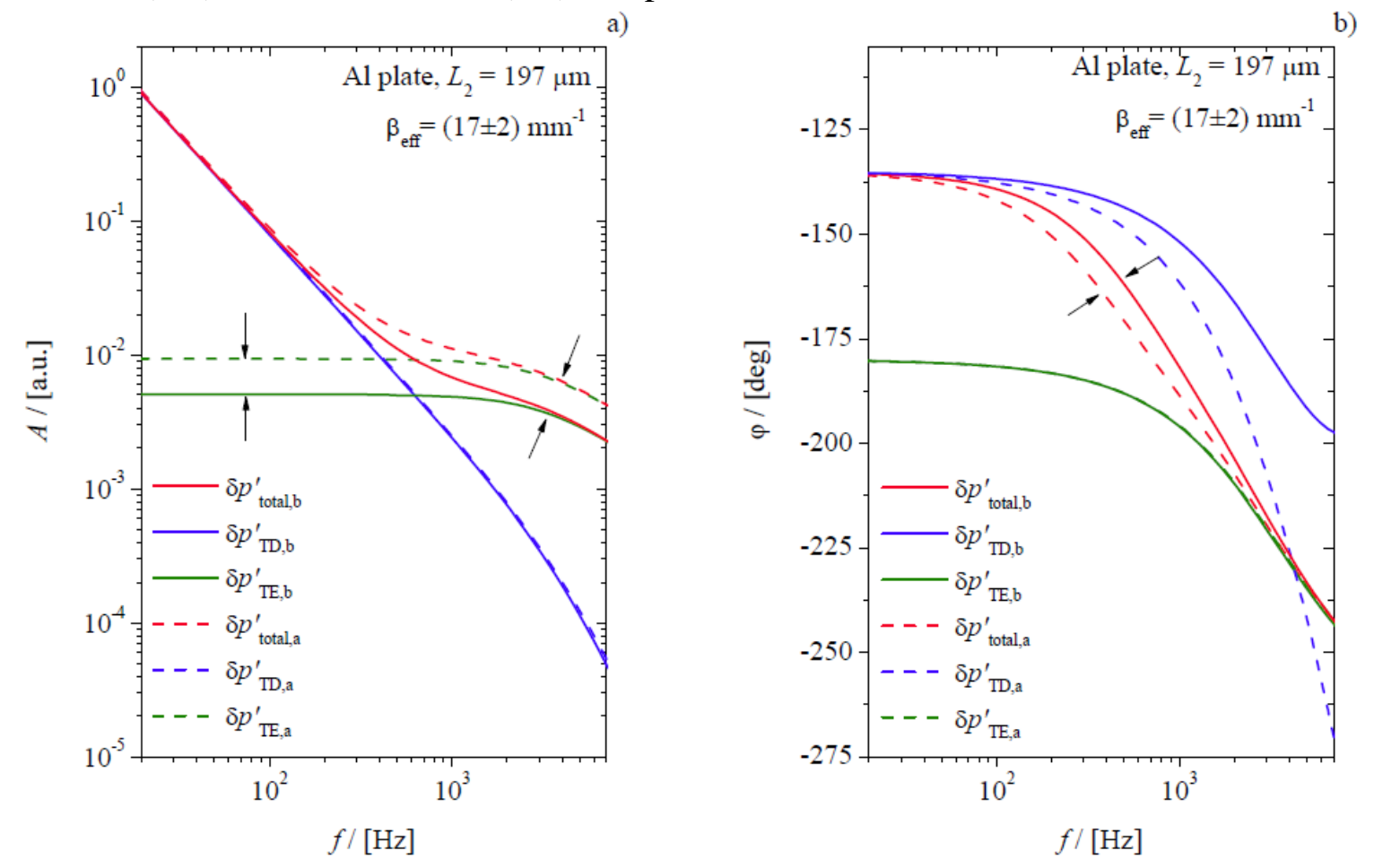

FIG. 7. The same than in Fig. (6), for $L_{2}=197 \mu \mathrm{m}$. 


\section{CONCLUSIONS}

The contributions of the thermal diffusion and thermoelastic bending of Al plates, on the open-cell photoacoustic signal have been experimentally and theoretically analyzed. This has been done by measuring the amplitude and phase of the photoacoustic signal, for modulation frequencies within the interval $20 \mathrm{~Hz}-7 \mathrm{kHz}$ and three $\mathrm{Al}$ samples with thicknesses of 280 $\mu \mathrm{m}, 197 \mu \mathrm{m}$, and $112 \mu \mathrm{m}$. The application of the standard thermoelastic theory for opaque materials has been extended to volume-absorber samples, with an effective absorption coefficient. Based on the comparison of the experimental data and these theoretical models, it has been shown that: 1) the "opaque" approach is not able to accurately describe the frequency dependence of the amplitude and phase of the photoacoustic signal. The observed deviations are strongly present for modulations frequencies higher than $200 \mathrm{~Hz}$, and they are larger in the amplitude than in the phase. 2) A quite good agreement between the volumeabsorber model and the experimental data for both the amplitude and phase is obtained in the whole frequency range, for the $280 \mu \mathrm{m}$ and $197 \mu \mathrm{m}$ thick samples. 3) For the $112 \mu \mathrm{m}$ thick plate, the new approach exhibits a slight deviation from the measured phase, due to its strong thermoeleastic bending, which is not taking into account in the theory. Within the approximation of the small bending, the proposed "volume-absorber" model extend the applicability of the classical "opaque" one, by providing an accurate description of the photoacoustic signal for Al samples thicker than $112 \mu \mathrm{m}$.

\section{ACKNOWLEDGMENTS}

This work was supported by the Ministry of Education, Science and Technological Development of the Republic of Serbia (projects ON171016 and III45005). 


\section{Appendix I. Volume-absorber Approximation ${ }^{18,19}$}

By considering the Al sample (Figure 1) as a volume-absorber material with an absorption coefficient $\beta$, the temperature distribution within the sample $T_{s}(x)$ (Fig. 1) can be calculated by solving the heat diffusion equation

$$
\frac{\partial^{2} T}{\partial x^{2}}-\frac{1}{D_{s}} \frac{\partial T}{\partial t}=-\frac{\beta I_{0}}{k_{s}} \exp [-\beta(x+L / 2)+j \omega t]
$$

whose general solution has the form $T(x, t)=T_{s}(x) \exp (j \omega t)$ and is given by

$$
T_{s}(x)=A_{1} \sinh \left(\sigma_{s}(L / 2-x)\right)+A_{2} \cosh \left(-\sigma_{s}(L / 2-x)\right)-A_{3} e^{-\beta(x+L / 2)},
$$

where $\sigma_{s}=\sqrt{j \omega / D_{s}}$ and the constant $A_{3}$ is given by

$$
A_{3}=\frac{\beta I_{0}}{k_{s}\left(\beta^{2}-\sigma_{s}^{2}\right)} .
$$

The constants $A_{1}$ and $A_{2}$ are obtained from the continuity of the heat flux at the boundaries $x=-L / 2 ; L / 2$. Taking into account that the thermal effusivity $\left({ }_{1}\right)$ of the Al sample is much greater than the one $\left(\varepsilon_{2}\right)$ of the surrounding air $\left(\varepsilon_{1}>>\varepsilon_{2}\right)$, these boundary conditions read

$$
\begin{aligned}
& \left.\frac{\partial T}{\partial x}\right|_{x=-L / 2}=0, \\
& \left.\frac{\partial T}{\partial x}\right|_{x=L / 2}=0 .
\end{aligned}
$$

The combination of Eqs. (A2)-(A5) yields

$$
\begin{aligned}
& A_{1}=\frac{\beta A_{3}}{\sigma_{s}} e^{-\beta L}, \\
& A_{2}=\frac{\beta A_{3}}{\sigma_{s}} \frac{1-e^{-\beta L} \cosh \left(\sigma_{s} L\right)}{\sinh \left(\sigma_{s} L\right)} .
\end{aligned}
$$

Finally, by inserting Eqs. (A3), (A6), and (A7) into Eq. (A2), one obtains

$$
T_{s}(x)=A_{3}\left\{\frac{\beta}{\sigma_{s}} \frac{\cosh \left[\sigma_{s}(L / 2-x)\right]-e^{-\beta L} \cosh \left[\sigma_{s}(L / 2+x)\right]}{\sinh \left(\sigma_{s} L\right)}-e^{-\beta(L / 2+x)}\right\} .
$$




\section{Appendix II. Effective absorption coefficient}

The intensity $I(x)$ of the laser at the position $x$ inside a material is determined by the beerlambert law, ${ }^{12,36}$ which establishes that

$$
I(x)=I_{0}(1-R) e^{-\beta x},
$$

where $I_{0}$ is the incident intensity on the surface $x=0, R$ is its reflectivity, and $\beta$ is the absorption coefficient of the material. This layer intensity represents an inner heat source $S(x)=\beta I(x)$. Applying Eq. (B1) to our (paint+Al) sample, which is a two-layer system, we obtain that the laser intensity $I_{2}$ inside the Al layer is given by

$$
I_{2}(x)=\left[I_{0}\left(1-R_{1}\right) e^{-\beta_{1} l_{1}}\right]\left(1-R_{2}\right) e^{-\beta_{2} x},
$$

where the subscripts 1 and 2 stand for the properties of the first (paint) and second (Al) layer, respectively. The rate of light-to-heat conversion inside the Al layer is then determined by the heat source $S_{2}(x)=\beta_{2} I_{2}(x)$, which by comparison with the heat source of a single layer, indicates that the effective absorption coefficient $\beta_{\text {eff }}$ of our (paint+Al) sample can be written as

$$
\beta_{\text {eff }}=\beta_{2}\left(1-R_{1}\right)\left(1-R_{2}\right) e^{-\beta_{1} l_{1}} .
$$

Taking into account the high reflectivity of $\mathrm{Al}\left(R_{2}>0.9\right)$ and high absorbance of the black paint $\left(\beta_{1} l_{1}>1\right)$, it is clear that effective absorption coefficient is smaller than the one of the Al plate $\left(\beta_{\text {eff }}<\beta_{2}\right)$. 


\section{References}

1 M. V. Marquezini, N. Cella, A. M. Mansanares, H. Vargas, and L. C. M. Miranda, Meas. Sci. Technol. 2, 396 (1991).

2 F. B. G. Astrath, N. G. C. Astrath, M. L. Baesso, A. C. Bento, J. C. S. Moraes, and A. D. Santos, J. Appl. Phys. 111, 014701 (2012).

3 J. A. Balderas-Lopez and A. Mandelis, J. Appl. Phys. 90, 2273 (2001).

4 A. M. Mansanares, A. C. Bento, H. Vargas, N. F. Leite, and L. C. M. Miranda, Phys. Rev. B 42, 4477 (1990).

5 H. Vargas and L. C. M. Miranda, Phys. Rep. 161, 43 (1988).

6 J. F. Mcclelland and R. N. Kniseley, Appl. Opt. 15, 2967 (1976).

7 A. Rosencwaig and S. S. Hall, Anal. Chem. 47, 548 (1975).

8 G. C. Wetsel and F. A. Mcdonald, IEEE T. Son. Ultrason. 25, 258 (1978).

9 A. Rosencwaig and A. Dermarderosian, J. Nat. Prod. 38, 544 (1975).

${ }^{10}$ P. Charpentier, F. Lepoutre, and L. Bertrand, J. Appl. Phys. 53, 608 (1982).

11 A. G. Bell, American Journal of Science 20, 305 (1880).

12 A. Rosencwaig and A. Gersho, J. Appl. Phys. 47, 64 (1976).

13 F. A. Mcdonald and G. C. Wetsel, J. Appl. Phys. 49, 2313 (1978).

14 E. Marin, H. Vargas, P. Diaz, and I. Riech, Phys. Status Solidi a-Appl. Res. 179, 387 (2000).

15 J. J. Alvarado-Gil, O. Zelaya-Angel, H. Vargas, and J. L. Lucio, Phys. Rev. B 50, 14627 (1994).

16 J. J. Alvarado-Gil, O. Zelaya-Angel, F. Sanchez-Sinencio, H. Vargas, and J. L. Lucio, Vacuum 46, 883 (1995).

17 J. L. Pichardo-Molina and J. J. Alvarado-Gil, J. Appl. Phys. 95, 6450 (2004).

18 M. D. Dramicanin, Z. D. Ristovski, P. M. Nikolic, D. G. Vasiljevic, and D. M. Todorovic, Phys. Rev. B 51, 14226 (1995).

19 D. M. Todorovic, P. M. Nikolic, M. D. Dramicanin, D. G. Vasiljevic, and Z. D. Ristovski, J. Appl. Phys. 78, 5750 (1995).

20 A. Mandelis and P. Hess, Semiconductors and electronic materials (SPIE Press, Bellingham, 2000).

${ }^{21}$ G. G. de la Cruz and Y. G. Gurevich, J. Appl. Phys. 80, 1726 (1996).

${ }^{22}$ G. Rousset, F. Lepoutre, and L. Bertrand, J. Appl. Phys. 54, 2383 (1983).

23 L. F. Perondi and L. C. M. Miranda, J. Appl. Phys. 62, 2955 (1987).

${ }^{24}$ S. Timoshenko and S. Woinowsky-Krieger, Theory of plates and shells, $2 \mathrm{~d}$ ed. (McGrawHill, New York, 1959).

25 A. W. Leissa, Vibration of plates (Scientific and Technical Information Division, National Aeronautics and Space Administration, Washington, 1969).

26 Y. X. Sun and M. Saka, J. Sound Vib. 329, 328 (2010).

27 A. Somer, F. Camilotti, G. F. Costa, C. Bonardi, A. Novatski, A. V. C. Andrade, V. A. Kozlowski, and G. K. Cruz, J. Appl. Phys. 114 (2013).

${ }^{28}$ M. D. Rabasovic, M. G. Nikolic, M. D. Dramicanin, M. Franko, and D. D. Markushev, Meas. Sci. Technol. 20, 095902 (2009).

${ }^{29}$ D.D. Markushev, M.D. Rabasović, D.M. Todorović, S. Galović, and S. E. Bialkowski, Rev. Sci. Instrum. 86, 035110 (2015). 
30 A. C. Bento, D. T. Dias, L. Olenka, A. N. Medina, and M. L. Baesso, Braz. J. Phys. 32, 483 (2002).

31 L. Nicolaides and M. Mandelis, J. Appl. Phys. 90, 1255 (2001).

32 R. C. Weast, CRC handbook of chemistry and physics (CRC Press, Boca Raton, FL, 1988).

33 A. Nayar, The metals databook (McGraw-Hill, New York, 1997).

${ }^{34}$ R. B. Ross, Metallic materials specification handbook, 4th ed. (Chapman \& Hall, London, New York, 1992).

35 A. D. Rakić, A. B. Djurišic, J. M. Elazar, and M. L. Majewski, Appl. Opt. 37, 5271 (1998).

36 D. P. Almond and P. M. Patel, Photothermal Science and Techniques (Chapman \& Hall, London, 1996). 